\title{
Spontaneous Symmetry Breaking in Data Visualization
}

\author{
Feldager, Cilie W.; Hauberg, Søren; Hansen, Lars Kai
}

Published in:

Artificial Neural Networks and Machine Learning

Link to article, DOI:

10.1007/978-3-030-86340-1_35

Publication date:

2021

Document Version

Peer reviewed version

Link back to DTU Orbit

\section{Citation (APA):}

Feldager, C. W., Hauberg, S., \& Hansen, L. K. (2021). Spontaneous Symmetry Breaking in Data Visualization. In I. Farkaš, P. Masulli, S. Otte, \& S. Wermter (Eds.), Artificial Neural Networks and Machine Learning (pp. 435446). Springer. Lecture Notes in Computer Science (including subseries Lecture Notes in Artificial Intelligence and Lecture Notes in Bioinformatics) Vol. 12892 LNCS https://doi.org/10.1007/978-3-030-86340-1_35

\section{General rights}

Copyright and moral rights for the publications made accessible in the public portal are retained by the authors and/or other copyright owners and it is a condition of accessing publications that users recognise and abide by the legal requirements associated with these rights.

- Users may download and print one copy of any publication from the public portal for the purpose of private study or research.

- You may not further distribute the material or use it for any profit-making activity or commercial gain

- You may freely distribute the URL identifying the publication in the public portal 


\title{
Spontaneous Symmetry Breaking in Data Visualization
}

\author{
Cilie W. Feldager ${ }^{1}$, Søren Hauberg ${ }^{1}$, and Lars Kai Hansen ${ }^{1}$ \\ Section for Cognitive Systems, Technical University of Denmark \\ \{cife, sohau, 1kai\}@dtu.dk
}

\begin{abstract}
Data visualization tools should create low-dimensional representations of data that emphasize structure and suppress noise. However, such non-linear amplifications of structural differences can have side effects like spurious clustering in t-SNE (Amid and Warmuth [1]). We present a more general class of spurious structure, namely broken symmetry, defined as visualizations that lack symmetry present in the underlying data. We develop a simple workflow for detection of broken symmetry and give examples of spontaneous symmetry breaking in t-SNE and other well-known algorithms such as GPLVM and kPCA. Our extensive, quantitative study shows that these algorithms frequently break symmetry, thereby highlighting new shortcomings of current visualization tools.
\end{abstract}

\section{Motivation}

Data visualization is a core tool in the machine learning toolbox. Data sets are visualized for exploration, to formulate hypotheses and to make modeling decisions. Visualization is commonly used for interpretation of learned models, e.g. visualization of latent variables of a generative model to understand representations. Data visualization is also very useful for debugging. For these applications faithfulness is a concern - can we trust the structure revealed in a visualization?

Most data of interest is high dimensional, hence can not be directly visualized. Rather, some form of dimensionality reduction is required, which inevitably will lead to loss of information. Popular schemes such as t-SNE [27], aim at two or three-dimensional representations that capture both local and global structure in data. Fig. 1 shows a two-dimensional t-SNE visualization of images from the COIL-20 dataset [20]; the given example concerns a wooden object on a turntable that is viewed from multiple, equidistant angles forming full $360^{\circ}$ rotation. Such an incremental physical rotation leads to a set of images with a simple topological structure which can be quantified by the neighborhood graph. More specifically, we form a graph with the images as nodes and connect neighboring nodes along the rotation path to obtain the graph of a circle. The neighborhood graph presents us with a strong physical symmetry and we naturally expect a visualization of the data to reveal this pattern by a structure which is topologically equivalent to a circle. Evidently, this does not happen: The visualization has broken the symmetry and "invented" a difference between neighboring points that is non-physical. 
The significance of transformations and the ensuing question of symmetry preservation goes beyond the physical rotations of the COIL data set. Parameterized transformations are key to modern data augmentation strategies. The question of preservation of symmetries in augmented data sets is then related to whether given symmetries are successfully represented during learning.

Our contribution is to identify a new, general class of spurious structures in data visualization, namely spontaneously broken symmetry, defined as representations that lack symmetry present in the underlying data. We provide a topological, quantitative measure to detect broken symmetry (Sec. 2) allowing for a systematic

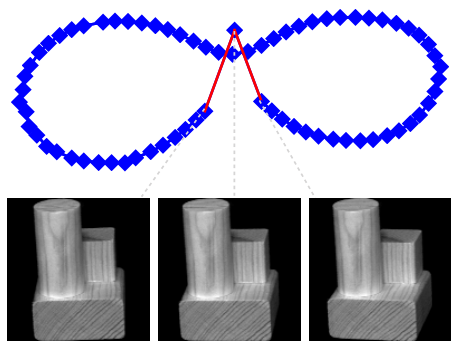

Fig. 1. We analyse a set of images of an object subject to a $360^{\circ}$ rotation on a turntable. The nearest neighbor graph forms a simple circle, however when the set is visualized using t-SNE the symmetry is lost.

study. Our empirical studies (Sec. 3) show that widely used visualization techniques break simple symmetries like rotations, hence, challenging the notion that they conserve global structure.

\section{Symmetries, Graphs, and Persistent Homology}

Symmetry groups. We consider symmetries, i.e. a property of a system that remains unchanged under a given transformation. The images of the wooden toy in Fig. 1 are formally equivariant when the toy is rotated physically on the turntable, while the outputs of a deep network for image based object classification ideally would be invariant (symmetric) under rotation.

Mathematically, such transformations and symmetries are described by Lie groups [11]. A real Lie group is a smooth differentiable manifold on which points are connected through a group operation and its inverse. For instance, rotation matrices form a smooth group with the matrix multiplication group operation. The unit circle can then be generated by a single unit vector

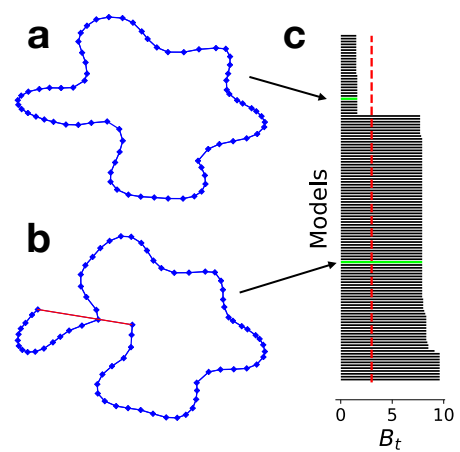

Fig. 2. The latent space of a model that preserves symmetry (a) and one that does not (b) (c) A barcode as a function of thresholds $B_{t}$. and its multiplication with all members of the group of rotation matrices. If the rotation group governs a physical phenomenon then we expect to observe data along a path that topologically is a circle, disregarding observation noise.

This paper focus on situations where the governing group is known and investigate if its structure is preserved by common visualization techniques. This is achieved by verifying if the group topology remains intact under visualizations.

Discrete approximations. In practice, we only observe a finite number of data points, rather than the entirety of a group. We can, however, approximate the 
path spanned by the observations with a graph, where points are connected if their generating group elements are close under the group metric. For instance, we may connect rotated images in a graph if their rotation angles are similar.

Measuring broken symmetry. For visualization, we map data to a low-dimensional space (typically $\mathbb{R}^{2}$ ); we let $X=\left\{x_{i}\right\}$ denote data coordinates in this lowdimensional space. We can now determine if a symmetry has been preserved under visualization by asking if the associated graph can be recovered from the low-dimensional coordinates. As the graph informs as to which points should be neighbors, we measure for each set of neighbors the radius of the ball needed to include one in the other's neighborhood graph. To compare across methods, we scale all distances by their median

$$
B_{\text {median }}=\operatorname{median}_{\left(x_{i}, x_{j}\right) \in G}\left(\left\|x_{i}-x_{j}\right\|\right),
$$

where $G$ denotes the graph associated with the generating group. We rely on the median due to its high breakdown point [15]. We, thus, measure

$$
B_{i j}=\frac{\left\|x_{i}-x_{j}\right\|}{B_{\text {median }}} .
$$

We can then threshold this measure such that, we say that a symmetry has been broken if $B_{i j}>B_{t}$ for any pair or equivalently, $\max \left(B_{i j}\right)>B_{t}$. We define $B_{\max }:=\max \left(B_{i j}\right)$. Note that this measure does not distinguish between one or multiple instances of broken symmetry.

Persistent homology. The measure above is linked with persistent homology [12]. This is a key mathematical tool in topological data analysis that has been shown to be robust to perturbations of the input data [6]. Following Carlsson [5], we place balls on each data point with radius $\epsilon$ and points falling within this ball defines a neighborhood. This defines a topological space $\Omega_{\epsilon}$. By varying $\epsilon$, we can create multiple topological spaces and let the Betti numbers $b_{i}\left(\Omega_{\epsilon}\right)$ quantify the structure of the topological space. The number $b_{0}$ represents the approximate number of connected components and $b_{1}$ the number of circles or holes.

In persistent homology, we study a spectrum of neighborhood sizes. For a known generating group, we would know its Betti numbers, and may ask which (if any) $\epsilon$ yield the given Betti numbers in the visualization point set. This allows us to consider multiple thresholds of our measure (2) of symmetry.

Barcodes. A broken symmetry is defined by the maximum of the normalized pairwise distances $B_{\max }$ being greater than a threshold $B_{t}$. This we can represent by a bar ranging from zero to $B_{\max }$ that visualizes the birth and death of symmetry. Stacking such bars (as in Fig. 2) yields a barcode. This lets us inspect the sensitivity of a chosen threshold for multiple models visually as each bar corresponds to a model [10]. The 'sharper' the transition from short bars to long bars is, the more robust the conclusion is. The barcode in Fig. 2 suggests that a 


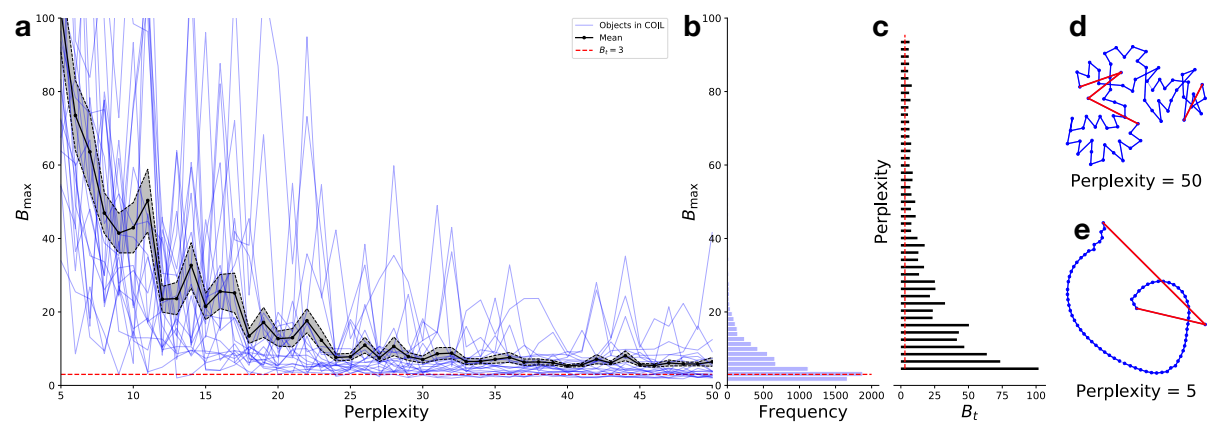

Fig. 3. (a) $B_{\max }$ vs. the perplexity for t-SNE. Each blue line represents the mean over 30 repeats for an object in COIL-20. The dotted, red line marks $B_{t}=3$ and the black lines represent mean and standard error over all objects. (b) Histogram of models. (c) Barcode for the mean (black lines in $(a)$ of objects. $(d)$ Latent space in model with perplexities 50 ( $B_{\max }$ is small). (e) Latent space in model with perplexities 5 ( $B_{\max }$ is large).

choice of $B_{t}=3$ is robust as any value in $B_{t} \in[2,8]$ yields the same conclusions. For quantitative comparisons across experiments we consistently use $B_{t}=3$ though this may be suboptimal for some models.

\section{$3 \quad$ Experiments}

We consider four methods representing the spectrum of visualization techniques:

t-SNE matches an exponential distribution of pairwise distances in data space with a t-distribution of pairwise distances in the latent space [27]. The visualization is controlled by a perplexity parameter that quantifies the effective number of neighbors used in the exponential distribution over pairwise distances. This is a randomized model as implemented in scikit learn [22].

TriMap [2] is a recent method that relies on an elaborate triplet weighting scheme such that point triplets are weighted with their pairwise distance before obtaining the final triplet weight $\omega_{i j k}=\zeta_{\gamma}\left(\delta+\tilde{\omega}_{i j k} / \omega_{\max }\right)$. Here $\zeta_{\gamma}(u)=\log (1+$ $\gamma u$ ), where the the locality parameter $\gamma$ is said to place focus on either local or global structure. The method is randomized and experiments were performed using software provided by Amid and Warmuth [2] where the default value is $\gamma=500$.

Kernel principle component analysis (kPCA) [25] extends classic PCA through the kernel trick. We use the squared exponential kernel $k\left(x_{i}, x_{j}\right)=$ $\exp \left(-\left\|x_{i}-x_{j}\right\|^{2} / \lambda\right)$, which is controlled by the scale parameter $\lambda$. The model is deterministic and experiments were performed using scikit learn [22].

Gaussian process latent variable model (GPLVM) [17] visualizes data using a latent representation with a Gaussian process prior with covariance function $k_{i j}=\theta \exp \left(-1 / 2\left\|x_{i}-x_{j}\right\|^{2}\right)+\sigma^{2} \delta_{i j}$, where $x_{i}, x_{j}$ denote latent points. 
The model is deterministic for a given initial condition of the hyperparameters $\theta$ and $\sigma^{2}, \theta_{0}$ and $\sigma_{0}^{2}$. Experiments were performed using Pyro [4].

In all experiments, we vary method parameters over a large range, and randomized methods are repeated multiple times and reported numbers are averages. Experimentally, we focus on the most elementary symmetry of interest: the rotation group. We consider images from (1) COIL-20 where objects are rotated $360^{\circ}$ in 72 steps and (2) MNIST where we synthetically rotate images with up to $360^{\circ}$ and $5 \%$ Gaussian noise is added to the pixel intensities. We perform a detailed analysis of each model's behavior, and quantitatively compare and summarize in Sec. 3.5.

\section{1 t-Distributed Stochastic neighborhood Embedding (t-SNE)}

To investigate possible symmetry breaking in t-SNE, we fit $30 \mathrm{t}$-SNE models to images of each COIL-20 object over a large span of perplexity parameters. We measure $B_{\max }=\max B_{i j}$ and report averages over the 30 models (the blue lines in Fig. 3a) ${ }^{1}$. As perplexity increase, $B_{\max }$ becomes smaller. This is to be expected as perplexity controls the smoothness of the t-SNE model. In $73 \%$ of all models, we observe broken symmetry $\left(B_{\max }>3\right)$. The barcodes reveal that this percentage is not particular sensitive to the choice of threshold (the red dotted line correspond to $B_{t}=3$ ). On MNIST, we observe a similar pattern (omitted due to space constraints) with $96.5 \%$ of all models having a broken symmetry.

In our experience, t-SNE tends to amplify small gaps in the data, leading to broken symmetry. This is linked to the 'spurious clustering' effect observed by Amid and Warmuth [1]. We generally observe that random initialization of t-SNE seems to better preserve symmetries than initialization by other methods such as PCA or Isomap. This former approach requires multiple restarts and choosing the embedding with lowest KL divergence.

\subsection{TriMap}

Amid and Warmuth [2] developed TriMap motivated by the spurious clustering effect in t-SNE, and we hypothesized that TriMap would lead to less symmetry breaking. However, the evidence in Fig. 4 does not support that conclusion. As before, each blue line shows the average $B_{\max }$ for 30 randomly initialized models for each object in COIL-20 over a wide span of the $\gamma$ parameter. Here $77 \%$ of all models are estimated to show broken symmetry, which is roughly on par with t-SNE. The barcode indicates that the choice of threshold is robust, though we find some inter-object variability (omitted). Our findings for MNIST are similar with $93.32 \%$ estimated symmetry breaking.

\subsection{Kernel Principal Component Analysis (kPCA)}

In $\mathrm{kPCA}$, we examine symmetries as a function of the kernel scale parameter $\lambda$. The barcode (Fig. 5) shows the robustness of the conclusion of preserved

\footnotetext{
${ }^{1} B_{\max }$ axis is cut off intentionally as the value for some object diverge
} 


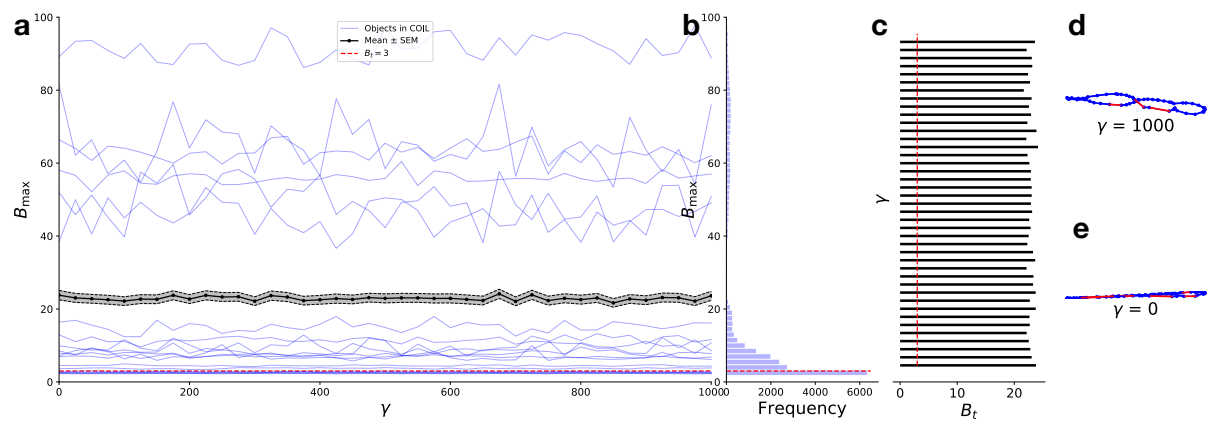

Fig. 4. (a) $B_{\max }$ vs. the locality parameter $\gamma$ for TriMap. Each blue line represents the mean over 30 repeats for an object in COIL-20. The dotted, red line marks $B_{t}=3$ and the black lines represent mean and standard error over all objects. (b) Histogram of models. (c) Barcode for the mean (black lines in $(a)$ of objects. $(d)$ Latent space in model with $\gamma=1000$. (e) Latent space in model with $\gamma=0$.

symmetry for the mean across COIL-20 objects. For large values of the scale parameter, the conclusion is robust as $B_{t}$ can vary, but for smaller values, our conclusions become sensitive to the specific choice of $B_{t}$.

In the non-linear regime (small values of $\lambda$ ), $B_{\text {median }}(1)$ is driven to small values (Fig. $5 \mathrm{~d}$ ) and $B_{\max }$ diverges. In the linear regime (large values of $\lambda$ ), the model approaches PCA which explains the flattening (Fig. 5e).

In $42 \%$ of models, we observe broken symmetry and note that five objects in COIL-20 give rise to broken symmetries: Object 2 (wooden toy), object 16 (round bottle), object 16 (ceramic vase), object 18 (tea cup) and object 20 (round container). Of these, four are rotationally symmetric in the plane of rotation, supporting our hypothesis that additional symmetry can induce symmetry breaking.

On MNIST data, the rate of broken symmetries was $7.23 \%$. One possible explanation for this reduction, is that if PCA on the MNIST data does not induce symmetry breaking then fewer models will break the symmetry because kPCA converges to $\mathrm{PCA}$ in the linear regime.

\subsection{Gaussian Process Latent Variable Model (GPLVM)}

We investigated the GPLVM design space by varying the initial values of the kernel hyperparameters, $\theta_{0}$ and $\sigma_{0}^{2}$ all with identical initialization of the latent space (isomap [26]). In Fig 6a, $\theta_{0}$ is fixed and $\sigma_{0}^{2}$ is varying and in Fig $6 \mathrm{~b}, \sigma_{0}^{2}$ is fixed while $\theta_{0}$ varies. An interesting thing to notice is while we mostly get consistent results, sometimes a small change in the initial condition induces a large change in the $B_{\max }$ leading to somewhat complex behavior.

The loss is often an indicator of broken symmetry as we saw with the KL divergence for t-SNE. If the parameter space contains symmetry-preserving models then these generally have lower loss than models that break symmetry. 


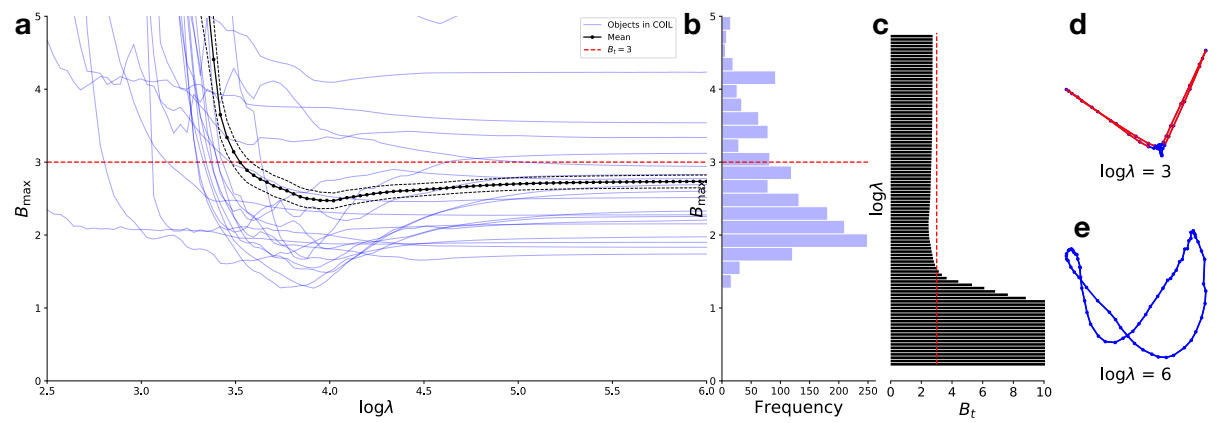

Fig. 5. (a) $B_{\max }$ vs. the scale parameter $\lambda$ for kPCA. Each blue line represents an object in COIL-20. The dotted, red line marks $B_{t}=3$ and the black lines represent mean and standard error over all objects. (b) Histogram of models. (c) Barcode for the mean of objects (black lines in $(a))$. (d) Latent space of model with $\log \lambda=3\left(B_{\max }\right.$ is large). (e) Latent space of model with $\log \lambda=6$ ( $B_{\max }$ is small).

The hyperparameters $\theta$ and $\sigma^{2}$ converge to the final values independent of the model preserving the symmetry. This means that the difference in loss between symmetry-preserving and symmetry-breaking models must be accounted for by the latent variables. It also means that it is not possible to detect a broken symmetry from the optimized hyperparameters but rather, one have to consider the latent variables to detect a broken symmetry.

Like in $\mathrm{kPCA}$, we find broken symmetries in the most symmetric objects. In the GPLVM, this is linked to the choice of initialization of the latent space. Overall, we found broken symmetries in $65.48 \%$ of the models and similarly in MNIST (46.32\%).

\subsection{Summary of experiments}

We found broken symmetry in all models with a high prevalence as summarized in Fig. 7. Note that we did not tune the parameters but varied important parameters across large ranges and used default parameters for others.

All objects in COIL-20 are indeed symmetric in data space according to our estimator. One may expect that high-level features may be less susceptible to broken symmetry than raw data. To investigate we extracted features using ResNet18 [13] and found no broken symmetries in the extracted features and no consistent, significant difference when looking at symmetry in the models trained on extracted features. We noticed that the most symmetric objects generally experienced more broken symmetry across models.

\section{Related Works}

Data visualization is important at many steps in the machine learning process. Visualization is used exploratively to form hypotheses [3], for understanding latent representations in supervised learning [8] and generative models [9]. 

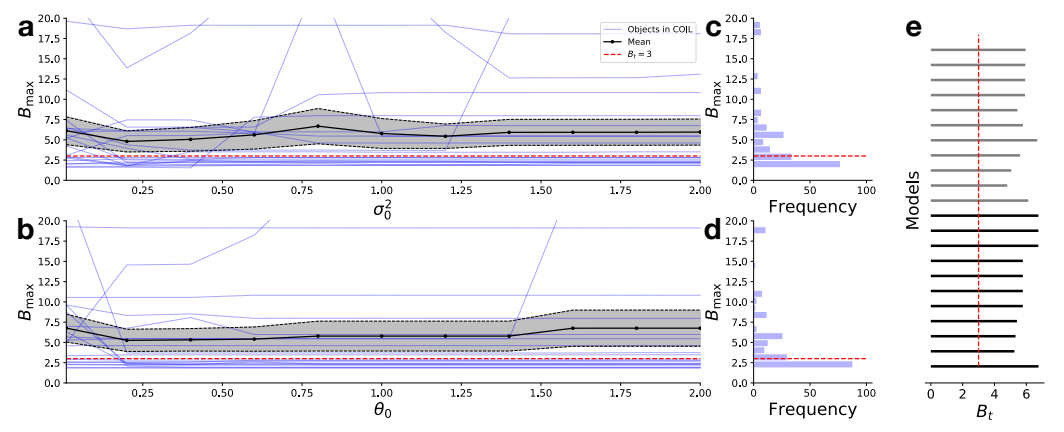

f

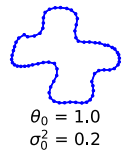

g

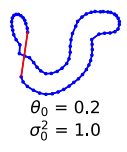

Fig. 6. (a) $B_{\max }$ vs. the initial value of the noise variance $\sigma_{0}^{2}$ for GPLVM. (b) $B_{\max }$ vs. the initial value of the kernel variance $\theta_{0}$ for GPLVM. Each blue line represents an object in COIL-20. The dotted, red line marks $B_{t}=3$ and the black lines represent mean and standard error over all objects. (a) Parameter space in $\sigma_{0}^{2}$ with fixed $\theta_{0}$. (b) Parameter space in $\theta_{0}$ with fixed $\sigma_{0}^{2}$. (c) Histogram of models in $a$. (d) Histogram of models in $b$. (e) Barcode for the mean of objects (black lines in $(a)$ and $(b))$. $(f)$ Latent space of model with $\theta_{0}=1$ and $\sigma_{0}^{2}=0.2$. $(g)$ Latent space of model with $\theta_{0}=0.2$ and $\sigma_{0}^{2}=0.2$.

The desiderata of visualization are discussed by Kaski et al. [16] and Venna et al. [28], who argue that visualizations should be trustworthy, meaning that samples appearing similar (e.g., neighbors) in the visualization should be similar in a physical sense. Also, they point out that data points close in a physical sense should be close in visualization. They noted the similarity with the concepts of precision and recall in information retrieval. Our concept of broken symmetry is related to the "recall" dimension, i.e., data that are physical neighbors, should also be visualized as such. The precision and recall criteria together measure the faithfulness of the visualization, see also Najim [19] for a related quantitative measure of the preservation of neighborhood relations in visualizations.

The immensely popular visualization scheme t-SNE [27] is constructed with the aim of representing both global and local structure. The original motivation for t-SNE included a critique of its predecessor SNE [14] for creating crowded visualizations, i.e., visualizations that did not show a clear separation of known clusters. Crowding is closely related to the trustworthiness concept of [16, 28]. By using a long-tailed distribution of the representations, t-SNE aims to fix the crowding problem. However, this emphasis of local dissimilarity comes at a price as noted in [18], simple manifolds like lines and sheets are broken apart in clusters. These clustering problems are examples of broken symmetry in our definition. Motivated by the problem of over-fitting cluster structure Amid and Warmuth [2] proposed TriMap. We observed, however, that TriMap cannot heal the problem of broken symmetry.

For detecting symmetries, we used topological data analysis [5], specifically persistent homology. Using this, we examined all values of thresholds simultaneously rather than study just a single threshold. Conveniently, Cohen-Steiner 

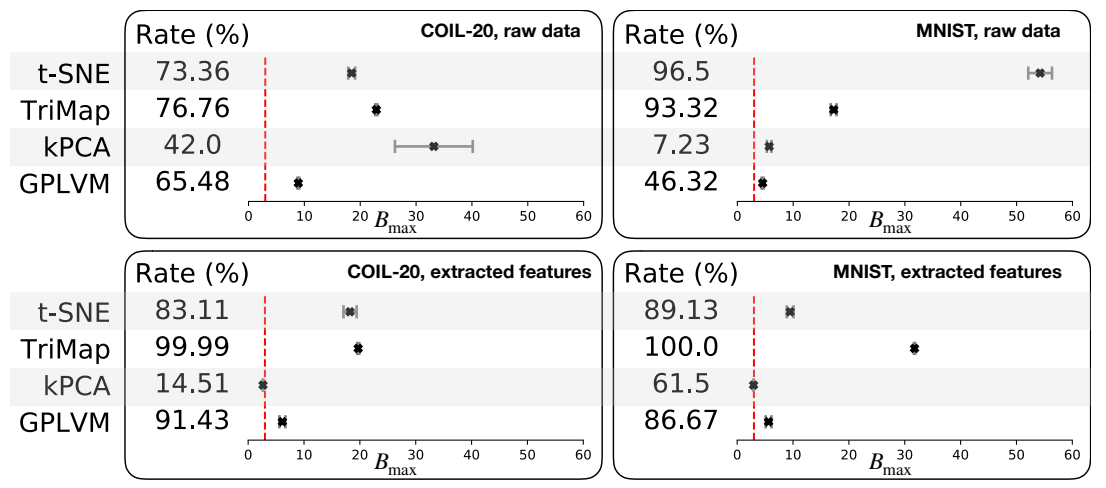

Fig. 7. Each panel shows the rate of broken symmetries in percent at $B_{t}=3$ with the mean and standard error plotted displayed on the axis for t-SNE, TriMap, kPCA, and GPLVM. Top, left pane) Summary of results on COIL-20. Top, right pane) Summary of results on MNIST. Bottom, left pane) Summary of results on features extract from COIL-20 using ResNet-18. Bottom, right pane) Summary of results on features extracted from MNIST using ResNet-18.

et al. [6] showed that the persistent homology tool is robust under pertubations of the data. [23] used persistent homology in its classical form whereas we have adapted it slightly as we knew which Betti numbers were required to preserve the symmetry. Our work exploits the coordinate and deformation invariances in topology and these properties aid in detecting symmetries as various deformations of the "circle" graph.

\section{Discussion}

We have investigated to which extend common visualization techniques are able to preserve simple symmetries, and have largely found the answer to be negative.

\subsection{Empirical findings}

We have investigated four popular algorithms that also represent different branches of the literature, namely t-SNE [27], TriMap [2], kPCA [25] and the GPLVM [17]. We have performed a systematic study of the influence of parameter choices in these methods by training more than 85.000 models over a wide parameter span. To quantitatively summarize these models' performance, we have introduced a simple scheme for detecting whether known symmetries are broken. Tools from persistent homology verify that this scheme is generally reliable, with some deviations for kPCA (see below).

t-SNE was found to be particularly sensitive to local optima and generally we found a need for multiple restarts. Fortunately, we generally observe that smaller KL reported values imply less symmetry breaking. Even with such mechanisms 
in place, we still see an overwhelming number of broken symmetries. Symmetry breaking can, to some extend, be reduced by increasing the perplexity parameter, but this also limits the flexibility and expressivity of the model.

TriMap, which was developed in part to alleviate problems with t-SNE, overall had comparable behavior to t-SNE with regards to broken symmetry. The $\gamma$ parameter, that controls the trade-off between capturing local or global structure, was found to have practically no effect with regards to symmetry breaking. We did not expect this, but have manually verified that broken symmetry is prevalent across large spans of $\gamma$.

kPCA was in a sense the most successful method according to our estimator. Kernel PCA, however, has a tendency to collapse points on to each other when mapping only two latent dimensions in the non-linear regime leading to strong symmetry breaking. On the other hand, $\mathrm{kPCA}$ reduces to conventional PCA in the limit of large kernel length scales, showing less symmetry breaking.

GPLVM was generally found to be sensitive to choice of initial parameters. While we have found it helpful to consider multiple restarts and choosing the model with highest likelihood, broken symmetries remain rather prevalent.

High-level features. One could suspect that symmetries are broken more commonly when working with raw data than with high-level abstract features, e.g., as those extracted by deep neural networks. We found no broken symmetries directly in the high-level features though when applying visualization algorithms, the prevalence was indeed high.

Summary. Our general finding is that symmetries are broken consistently across the studied methods. It is generally possible to manually tweak parameters to enforce that a known symmetry remains intact, but such strategies are not possible when the symmetry is unknown ${ }^{2}$, e.g. for knowledge discovery. We also note that default parameters of publicly available implementations of the studied methods generally perform poorly with regards to broken symmetry.

\subsection{Faithful representations}

At the heart of our study is the quest for faithful representations, i.e. representations that reflect the underlying physics of the data generating process. These have wider applicability than just visualization as studied here. For instance, a representation that is not faithful will most likely not result in a fair prediction. A broken symmetry can be viewed as model that violates the Lipschitz continuity condition. Individual fairness [7] can then no longer be ensured as similar individuals should be treated similarly.

Similar statements can be made for interpretable models, where 'almost discontinuous' models are generally difficult to interpret. From a purely predictive point of view, it is strictly not required that representations are faithful, though there is some evidence in that direction [24].

\footnotetext{
${ }^{2}$ It should be emphasized that while we consider known symmetries, we only do so in order to make quantitative statements.
} 
Finally, we note that visualization may be particularly sensitive to symmetry breaking as we tend to embed onto $\mathbb{R}^{2}$. While it is well-known that only few graphs (namely the planar ones) can be embedded in $\mathbb{R}^{2}$, then all graphs can be embedded in $\mathbb{R}^{3}[21]$. This suggests that symmetries are likely to be broken when data is forced onto a two-dimensional view (as is often the case in visualization), and indeed our experiments indicate that symmetry breaking is less frequent when embedding into three or more dimensions (omitted due to space constraints).

\subsection{Concluding remarks}

We have here pointed to a previously unnoticed problem in visualizations, namely broken symmetries. Through a systematic study of more than 85.000 trained models, we have found an alarming rate at which even the most simple symmetries are spontaneously broken during data visualizations. This suggest a need for both new methods that can reliably visualize high-dimensional data, but also for more systematic and quantitative evaluations of visualization techniques.

We have purposefully not investigated more complex symmetries as these raise complications that are beyond existing techniques; for instance, the twodimensional torus is mathematically impossible to embed in $\mathbb{R}^{2}$ without breaking the underlying symmetry. This calls for visualization techniques that embed onto curved surfaces in order to preserve symmetries, just as we use a sphere when we visualize global geoinformatics patterns.

Acknowledgements. This work received funding from the European Research Council (ERC) under the European Union's Horizon 2020 research and innovation programme (757360). SH were supported in part by a research grant (15334) from VILLUM FONDEN.

\section{References}

1. Amid, E., Warmuth, M.K.: A more globally accurate dimensionality reduction method using triplets. arXiv:1803.00854 [cs] (Mar 2018)

2. Amid, E., Warmuth, M.K.: TriMap: Large-scale Dimensionality Reduction Using Triplets. arXiv:1910.00204 [cs, stat] (Oct 2019)

3. Arora, S., Hu, W., Kothari, P.K.: An analysis of the t-sne algorithm for data visualization. arXiv preprint arXiv:1803.01768 (2018)

4. Bingham, E., Chen, J.P., Jankowiak, M., Obermeyer, F., Pradhan, N., Karaletsos, T., Singh, R., Szerlip, P., Horsfall, P., Goodman, N.D.: Pyro: Deep universal probabilistic programming. The Journal of Machine Learning Research 20(1), 973-978 (Jan 2019)

5. Carlsson, G.: Topology and data. Bulletin of the American Mathematical Society 46(2), 255-308 (Jan 2009)

6. Cohen-Steiner, D., Edelsbrunner, H., Harer, J.: Stability of Persistence Diagrams. Discrete \& Computational Geometry 37(1), 103-120 (Jan 2007)

7. Dwork, C., Hardt, M., Pitassi, T., Reingold, O., Zemel, R.: Fairness Through Awareness. arXiv:1104.3913 [cs] (Nov 2011) 
8. Esteva, A., Kuprel, B., Novoa, R.A., Ko, J., Swetter, S.M., Blau, H.M., Thrun, S.: Dermatologist-level classification of skin cancer with deep neural networks. Nature 542(7639), 115-118 (2017)

9. Frid-Adar, M., Diamant, I., Klang, E., Amitai, M., Goldberger, J., Greenspan, H.: Gan-based synthetic medical image augmentation for increased cnn performance in liver lesion classification. Neurocomputing 321, 321-331 (2018)

10. Ghrist, R.: Barcodes: The persistent topology of data. Bulletin of the American Mathematical Society 45(1), 61-75 (2008)

11. Hall, B.C.: Lie Groups, Lie Algebras, and Representations: An Elementary Introduction. Graduate Texts in Mathematics, Springer International Publishing, second edn. (2015)

12. Hatcher, A.: Algebraic topology. Cambridge University Press (2005)

13. He, K., Zhang, X., Ren, S., Sun, J.: Deep Residual Learning for Image Recognition. arXiv:1512.03385 [cs] (Dec 2015)

14. Hinton, G.E., Roweis, S.T.: Stochastic neighbor embedding. In: Advances in Neural Information Processing Systems. pp. 857-864 (2003)

15. Huber, P.J.: Robust statistics, vol. 523. John Wiley \& Sons (2004)

16. Kaski, S., Nikkilä, J., Oja, M., Venna, J., Törönen, P., Castrén, E.: Trustworthiness and metrics in visualizing similarity of gene expression. BMC bioinformatics 4, 48 (Oct 2003)

17. Lawrence, N.D.: Probabilistic Non-linear Principal Component Analysis with Gaussian Process Latent Variable Models. Journal of Machine Learning Research p. 34 (2005)

18. Linderman, G.C., Steinerberger, S.: Clustering with t-SNE, provably. arXiv:1706.02582 [cs, stat] (Jun 2017)

19. Najim, S.A.: Information visualization by dimensionality reduction: a review. Journal of Advanced Computer Science \& Technology 3(2), 101 (2014)

20. Nene, S.A., Nayar, S.K., Murase, H.: Columbia Object Image Library (COIL20). Technical Report CUCS-006-96 p. 6 (1996)

21. Nishizeki, T., Chiba, N.: Planar graphs: Theory and algorithms. Elsevier (1988)

22. Pedregosa, F., Varoquaux, G., Gramfort, A., Michel, V., Thirion, B., Grisel, O., Blondel, M., Prettenhofer, P., Weiss, R., Dubourg, V., Vanderplas, J., Passos, A., Cournapeau, D., Brucher, M., Perrot, M., Duchesnay, E.: Scikitlearn: Machine learning in Python. Journal of Machine Learning Research 12, 2825-2830 (2011)

23. Pokorny, F.T., Kjellström, H., Kragic, D., Ek, C.: Persistent Homology for Learning Densities with Bounded Support. In: Pereira, F., Burges, C.J.C., Bottou, L., Weinberger, K.Q. (eds.) Advances in Neural Information Processing Systems 25. pp. 1817-1825. Curran Associates, Inc. (2012)

24. Rieger, L., Singh, C., Murdoch, W.J., Yu, B.: Interpretations are useful: penalizing explanations to align neural networks with prior knowledge. arXiv preprint arXiv:1909.13584 (2019)

25. Schölkopf, B., Smola, A., Müller, K.R.: Nonlinear Component Analysis as a Kernel Eigenvalue Problem. Neural Computation 10(5), 1299-1319 (1998) 
26. Tenenbaum, J.B.: A Global Geometric Framework for Nonlinear Dimensionality Reduction. Science 290(5500), 2319-2323 (Dec 2000)

27. van der Maaten, L., Hinton, G.: Visualizing Data using t-SNE. Journal of machine learning research pp. 2579-2605 (2008)

28. Venna, J., Peltonen, J., Nybo, K., Aidos, H., Kaski, S.: Information Retrieval Perspective to Nonlinear Dimensionality Reduction for Data Visualization. Journal of Machine Learning Research 11(13), 451-490 (2010) 